TRANSACTIONS OF THE

AMERICAN MATHEMATICAL SOCIETY

Volume 354, Number 2, Pages 795-806

S 0002-9947(01)02822-7

Article electronically published on September 19, 2001

\title{
TRANSVERSE SURFACES AND ATTRACTORS FOR 3-FLOWS
}

\author{
W. J. COLMENAREZ AND C. A. MORALES
}

\begin{abstract}
We prove that a hyperbolic strange attractor of a three-dimensional vector field is a suspension if it exhibits a transverse surface over which the unstable manifold induces a lamination without closed leaves. We also study the topological equivalence of singular attractors exhibiting transverse surfaces for three-dimensional vector fields.
\end{abstract}

\section{INTRODUCTION}

In this paper we consider hyperbolic strange attractors with a transverse surface for three-dimensional vector fields. The motivation is the known fact that an Anosov three-dimensional vector field is a suspension if it exhibits a transverse torus over which the unstable manifold induces a foliation without closed leaves [Fe1], [Fe2]. Indeed, we generalize this result for hyperbolic strange attractors exhibiting transverse surfaces.

We are also interested in the topological equivalence of singular attractors exhibiting transverse surfaces for three-dimensional vector fields. Indeed, we show that there is no equivalence between the geometric Lorenz attractors [GW] and the singular attractor described in $\S 5$ (see Figure 2(a)). This result is claimed in MP], but the proof there is only a sketch. Here we fill in the details of the proof in MP by using the ideas developed in $\S 2$. We hope that similar ideas can be used to study the equivalence between singular attractors with transverse surface in a general setting.

Let us state our results precisely. Let $X$ denote a vector field on a closed manifold $M$ and let $X_{t}$ denote the flow generated by $X$. Define $\omega_{X}(q)$, the $\omega$-limit set of $q$, as the set of accumulation points of the positive orbit $\left\{X_{t}(q): t \geq 0\right\}$ of $q$. A compact invariant set $\Lambda$ of $X$ is transitive if $\Lambda=\omega_{X}(q)$ for some $q \in \Lambda$; singular if it contains a singularity of $X$; and attracting if it can be realized as $\bigcap_{t \geq 0} X_{t}(U)$ for some positively invariant open set $U$ (i.e. $X_{t}(U) \subset U, \forall t>0$ ). An attractor is a transitive attracting set.

An invariant set $\Lambda$ is hyperbolic if it has an invariant splitting $E^{s} \oplus E^{X} \oplus E^{u}$ so that $E^{s}$ is contracting, $E^{u}$ is expanding and $E^{X}$ is the direction of $X([\overline{\mathrm{PS}}])$. If $p \in \Lambda$, there are invariant manifolds $W_{X}^{s}(p), W_{X}^{u}(p), W_{X}^{s s}(p)$ and $W_{X}^{u u}(p)$ which are tangent respectively to $E_{p}^{s} \oplus E_{p}^{X}, E_{p}^{X} \oplus E_{p}^{u}, E_{p}^{s}$ and $E_{p}^{u}$ ([HPS]). The manifold

Received by the editors October 27, 1999 and, in revised form, November 15, 2000.

2000 Mathematics Subject Classification. Primary 37Dxx; Secondary 37C15.

Key words and phrases. Anosov flow, hyperbolicity, cross-section.

Partially supported by FAPERJ, CNPq and PRONEX of Brasil.

(C)2001 American Mathematical Society 
$W_{X}^{s s}(p)\left(W_{X}^{u u}(p)\right)$ is called a strong stable (unstable) manifold of $p$. It follows that $W_{X}^{s s}(p)=\left\{x \in M: d\left(X_{t}(x), X_{t}(p)\right) \rightarrow 0, t \rightarrow \infty\right\}$

and

$$
W_{X}^{u u}(p)=\left\{x \in M: d\left(X_{t}(x), X_{t}(p)\right) \rightarrow 0, t \rightarrow-\infty\right\} .
$$

In addition,

$$
W_{X}^{s}(p)=\bigcup_{t \in \mathbb{R}} W_{X}^{s s}\left(X_{t}(p)\right)
$$

and

$$
W_{X}^{u}(p)=\bigcup_{t \in \mathbb{R}} W_{X}^{u u}\left(X_{t}(p)\right) .
$$

A periodic orbit (singularity) of $X$ is hyperbolic if it is a hyperbolic set of $X$. If $\mathcal{O}$ is a hyperbolic periodic orbit of $X$, we denote $W_{X}^{s}(\mathcal{O})=W_{X}^{s}(p)$ for some $p \in \mathcal{O}$. Clearly, this definition does not depend on $p \in \mathcal{O}$. Similarly, $W_{X}^{u}(\mathcal{O})=W_{X}^{u}(p)$. A periodic orbit is attracting (repelling) if it is hyperbolic and $E^{u}=0\left(E^{s}=0\right)$. Similarly, for singularities. We say that the point $p \in M$ is periodic if its full orbit $\left\{X_{t}(p): t \in \mathbb{R}\right\}$ is a periodic orbit of $X$. In that case $p$ is hyperbolic if its corresponding periodic orbit is hyperbolic. The vector field $X$ is Anosov if $M$ is a hyperbolic set of $X$.

By a surface we mean a connected embedded 2-manifold with or without boundary. A surface $S$ is transverse to $\Lambda$ if (1) $X(q)$ is not tangent to $S(\forall q \in S)$ and (2) $\Lambda \cap S$ is nonempty and does not intersect the boundary of $S$ (if any). Following [Bo], we say that $\Lambda$ is a suspension if it exhibits a transverse surface intersecting every orbit of $X$ in $\Lambda$. We shall denote by $\operatorname{dim}(E)$ the dimension of a linear space E.

If $\Lambda$ is a hyperbolic attracting set, $\operatorname{dim}\left(E^{u}\right)=1$ and $S$ is a transverse surface of $\Lambda$, there is a nonsingular lamination $\mathcal{F}_{S}^{u}=\left\{W_{X}^{u}(p) \cap S\right\}_{p \in \Lambda \cap S}$ on $\Lambda \cap S$. This lamination is defined in particular for hyperbolic strange attractors, i.e. hyperbolic attractors with $\operatorname{dim}\left(E^{u}\right)=1$.

Theorem A. A hyperbolic strange attractor of a three-dimensional vector field is a suspension if it exhibits a transverse surface $S$ such that $\mathcal{F}_{S}^{u}$ has no closed leaves.

Observe that this result follows from [Fe1] if the attractor is $M$ because, in that case, the vector field is Anosov and the transverse surface is incompressible. Theorem A is proved without using the lifting flow to the universal cover; it will be a direct consequence of Theorem B in $\S 4$ (see Example 2.1). Our approach will be used in $\S 5$ to prove that the singular attractor in Figure 2(a) and the geometric Lorenz attractor in [GW] are not equivalent.

The second author thanks Pedro Duarte for helpful conversation.

\section{Preliminaries}

To begin, let $\Lambda$ be a compact invariant set of a three-dimensional vector field $X$. We say that $\Lambda$ is isolated if it can be realized as $\bigcap_{t \in \mathbb{R}} X_{t}(U)$ for some open set $U$. Obviously, attracting sets are isolated and the converse is not true.

We say that $\Lambda$ is connected if it cannot be realized as the disjoint union of two relatively closed nonempty subsets. It is immediate that transitive sets for flows are connected. 
If $S$ is a surface transverse to $\Lambda$, we define

$$
\sigma_{S}=\left\{q \in \Lambda: X_{t}(q) \notin S, \forall t \in \mathbb{R}\right\} \quad \text { and } \quad l_{S}=\left\{q \in \Lambda \cap S: X_{t}(q) \notin S, \forall t>0\right\} .
$$

Note that $\omega_{X}(q) \subset \sigma_{S}$ for any $q \in l_{S}$ since the orbits in $\Lambda$ do not intersect the boundary of $S$.

Following $[\overline{\mathrm{Fr}}]$, we say that $S$ is a cross-section of $\Lambda$ if $\sigma_{S}=\emptyset$. Observe that $\Lambda$ is a suspension if it exhibits a cross-section.

Example 1.0. The Lorenz attractor is an example of a nonhyperbolic singular attractor $\Lambda$ of a vector field $X$ exhibiting a transverse surface $S$ with $\sigma_{S} \neq \emptyset$. In this case, $\sigma_{S}$ is a saddle singularity and $l_{S}$ is a cantor set contained in a curve $c \subset W_{X}^{s}\left(\sigma_{S}\right) \cap S$. See Figure 2-(b).

Example 1.1. The supporting manifold of the Anosov flow in [BL] is an example of a hyperbolic attractor $\Lambda$ exhibiting a transverse surface. In this case, $S$ is a torus, $\sigma_{S}$ is a saddle periodic orbit and $l_{S}$ is the disjoint union of two freely homotopic closed curves in $S$.

Observe that, in the previous examples, $\Lambda$ is connected and $\sigma_{S}$ is not an attracting set. It seems that this is always true, namely $\sigma_{S}$ is not an attracting set for surfaces $S$ transverse to connected isolated sets. We shall prove this assertion below in the case where the involved isolated set is hyperbolic and attracting.

Theorem 1.0. If $S$ is a surface transverse to a hyperbolic connected attracting set, then $\sigma_{S}$ is not an attracting set.

The next example shows that the above theorem fails for surfaces transverse to compact connected invariant sets in general.

Example 1.2. Let $X$ be a three-dimensional vector field as in Figure 1 having a hyperbolic saddle periodic orbit $p$, a cross-section $S$ of $p$, an attracting singularity $\sigma$ and a flowline $\gamma$ in the unstable manifold of $p$ joining $p$ with $\sigma$ (here $l_{S}$ reduces to the point $q$ in Figure 1). Then, $\Lambda=p \cup \sigma \cup \gamma$ is a compact connected invariant (nonisolated) set exhibiting a transverse surface $S$ with $\sigma_{S}=\sigma$ attracting.

The hyperbolicity of the attracting set is used in the proof of Theorem 1.0 to obtain stable and unstable manifolds. The idea of the proof is as follows. By contradiction, assume that $S$ is a surface transverse to a connected hyperbolic attracting set $\Lambda$ and that $\sigma_{S}$ is an attracting set. Then $\sigma_{S} \neq \emptyset$, and so, $l_{S} \neq \emptyset$ (Lemma 1.2). Taking $q \in l_{S}$, by the Local Structure Product for hyperbolic sets, we shall have a situation which is similar to Figure 1, namely the backward orbit $\gamma$ of $q$ converges to the periodic point $p$. In particular, the unstable manifold of $p$ intersects $l_{S}$. By Lemma 1.3, since $\Lambda$ is an attracting set, there would exist a $q^{*} \in l_{S}$ and a connected arc $J^{*}$ in $\Lambda \cap S$ joining $q^{*}$ to some point in the orbit of $p$ (note that $q=q^{*}$ in Figure 1 ). This fact together with Lemma 1.1 would imply that $p \in \sigma_{S}$, a contradiction (see Corollary 1.4).

Let us present the details. In what follows, $\Lambda$ denotes an isolated set of a vector field $X$ and $S$ is a surface transverse to $\Lambda$.

Lemma 1.1. The set $\sigma_{S}$ is isolated. If $\sigma_{S}$ is an attracting set, then $l_{S}$ is open and closed in $\Lambda \cap S$.

Proof. The first claim is obvious. To prove the second one, observe that $l_{S}$ is closed in $\Lambda \cap S$ and $\omega_{X}(q) \subset \sigma_{S}$ for every $q \in l_{S}$. In particular, $l_{S}$ is open in $\Lambda \cap S$ if $\sigma_{S}$ is an attracting set. 


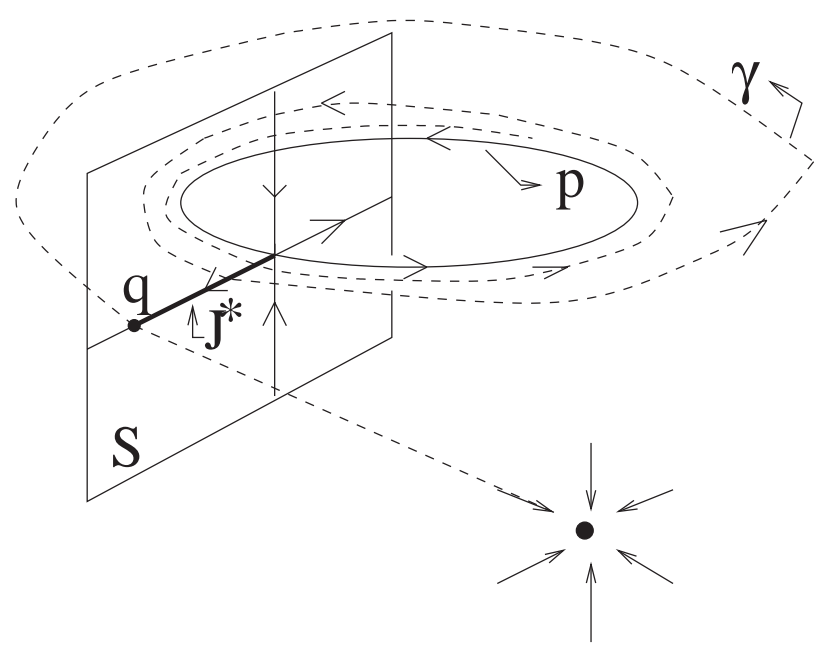

$\sigma$

FIGURE 1.

Clearly, $\sigma_{S}=\emptyset$ implies $l_{S}=\emptyset$. The converse fails in general as follows: Let $\Lambda$ be the disjoint union of two compact invariant sets, one of them suspended (with cross-section $S$ ). Denote the nonsuspended set by $\sigma$. Then, $S$ is a transverse surface of $\Lambda$, with $l_{S}=\emptyset$, and $\sigma_{S}=\sigma \neq \emptyset$. Note that $\Lambda$ is not connected in this case.

Lemma 1.2. If $\Lambda$ is connected and $l_{S}=\emptyset$, then $\sigma_{S}=\emptyset$.

Proof. By way of contradiction, assume that $\sigma_{S} \neq \emptyset$. Note that $\sigma_{S}$ is closed in $\Lambda$ by Lemma 1.1 .

As $l_{S}=\emptyset$ by hypothesis, for every $q \in \Lambda \cap S$ there is a first $t_{q}>0$ so that $X_{t_{q}}(q) \in S$. By the Tubular Flow-Box Theorem, as $\Lambda \cap S$ is compact, there is $\beta>0$ so that $t_{q} \leq \beta$ for all $q \in \Lambda \cap S$.

Let

$$
\Lambda^{*}=\bigcup_{q \in \Lambda \cap S}\left\{X_{t}(q): t \in\left[0, t_{q}\right]\right\} .
$$

Clearly, $\sigma_{S} \cap \Lambda^{*}=\emptyset$. We claim that $\Lambda^{*}$ is closed in $\Lambda$. Therefore, we choose a sequence $q_{n}^{\prime} \in \Lambda^{*}$ converging to $q \in \Lambda$. Then, by definition, there are sequences $q_{n} \in \Lambda \cap S$ and $t_{n} \in\left[0, t_{q_{n}}\right]$ so that $X_{t_{n}}\left(q_{n}\right)=q_{n}^{\prime} \rightarrow q$ as $n \rightarrow \infty$. Note that the sequence $t_{n}$ is bounded by $\beta$ since $t_{q_{n}}$ does. Then, using the compactness of $\Lambda \cap S$ and $[0, \beta]$, we can assume that $q_{n} \rightarrow q^{0} \in \Lambda \cap S$ and $t_{n} \rightarrow t^{0} \in[0, \beta]$. Thus, $X_{t^{0}}\left(q^{0}\right)=q$ by continuity of $X$. So $q \in \Lambda^{*}$ and the claim holds. Now the result follows from the claim since $\Lambda=\Lambda^{*} \cup \sigma_{S}$ is connected and both $\sigma_{S}$ and $\Lambda^{*}$ are closed and disjoint in $\Lambda$.

We denote $\mathcal{O}_{X}(p)=\left\{X_{t}(p): t \in \mathbb{R}\right\}$ as the orbit of $p$. Recall that $p \in M$ is a (hyperbolic) periodic point of $X$ if $\mathcal{O}_{X}(p)$ is a (hyperbolic) periodic orbit of $X$.

Lemma 1.3. Suppose that $\Lambda$ is an attracting set. If $p \in \Lambda \backslash \sigma_{S}$ is a hyperbolic periodic point and $W_{X}^{u}(p) \cap l_{S} \neq \emptyset$, then there is $q^{*} \in l_{S}$ and a compact connected curve $J^{*} \subset \Lambda \cap S$ joining $q^{*}$ to some $p^{\prime} \in \mathcal{O}_{X}(p)$. 
Proof. Clearly, $W_{X}^{u}(p) \neq \mathcal{O}_{X}(p)$ and $\mathcal{O}_{X}(p) \cap S \neq \emptyset$ since $p \notin \sigma_{S}$. Then, we can choose $p^{\prime} \in \mathcal{O}_{X}(p) \cap S$ and a path-connected relatively open set $I$ of $W_{X}^{u}(p) \cap S$ containing $p^{\prime}$.

Let

$$
\Pi: \operatorname{Dom}(\Pi) \subset S \rightarrow S
$$

be the return map of $X$ in $S$. Then, $p^{\prime} \in \operatorname{Dom}(\Pi)$ is $\Pi$-periodic with period $T$ (say). Shrinking $I$ and replacing $\Pi$ by $\Pi^{T}$ if necessary, we can assume that $I \subset \operatorname{Dom}(\Pi)$ and that $p^{\prime}$ is fixed.

Now, choose $\bar{q}_{0} \in W_{X}^{u}(p) \cap l_{S} \neq \emptyset$ by hypothesis. As the $\alpha$-limit set $\alpha_{X}\left(\bar{q}_{0}\right)=$ $\omega_{-X}\left(\bar{q}_{0}\right)$ is $\mathcal{O}_{X}(p)$, there is a sequence $q_{k} \in W_{X}^{u}(p) \cap S$ converging to $p^{\prime}$ so that $q_{0}=\bar{q}_{0}, q_{k} \in \operatorname{Dom}(\Pi)$ and $\Pi\left(q_{k}\right)=q_{k-1}$ for all $k \geq 1$. In particular, there is $n$ large so that $q_{n}, q_{n+1} \in I$.

Let $F_{n}$ be a connected curve in $I$ joining $q_{n}$ with $q_{n+1}$. This curve exists since $I$ is path connected. As $F_{n} \subset I \subset \operatorname{Dom}(\Pi)$, one can define inductively $F_{k}=\Pi\left(F_{k+1}\right)$, for $k \in\{0,1, \ldots, n-1\}$, as long as $F_{k+1} \in \operatorname{Dom}(\Pi)$.

Let $k^{*}$ be the smallest $k \in\{0,1, \ldots, n-1\}$ so that $F_{k} \subset \operatorname{Dom}(\Pi)$ and

$$
J=F_{n} \cup F_{n-1} \cup \ldots \cup F_{k^{*}+1} \cup F_{k^{*}} \text {. }
$$

Observe that $J \subset \Lambda \cap S$ by the flow invariance of $W_{X}^{u}(p)$ and the definition of $\Pi$ (recall that $\Lambda$ is an attracting set). Moreover, $J$ is a connected curve joining $q_{k^{*}}$ to $q_{n+1}$ since $\Pi\left(q_{k}\right)=q_{k-1}$ for all $k$.

Using the pathwise connectedness of $I$ and the fact that $p^{\prime} \in I$, we can joint $p^{\prime}$ to $q_{n+1}$ with a curve $J^{\prime} \subset I$. Define $J^{*}=J \cup J^{\prime}$. Then $J^{*}$ is a connected curve in $W_{X}^{u}(p) \cap S$. Clearly, $J^{*} \cap l_{S} \neq \emptyset$, and so, we can assume that $J^{*}$ has a boundary point $q^{*} \in l_{S}$ since $l_{S}$ is closed and satisfies $l_{S}=(\Lambda \cap S) \backslash \operatorname{Dom}(\Pi)$. Then, $J^{*} \subset \Lambda \cap S$ joints $q^{*} \in l_{S}$ to $p^{\prime} \in \mathcal{O}_{X}(p)$ proving the result.

Corollary 1.4. If both $\Lambda$ and $\sigma_{S}$ are attracting sets and $p \in \Lambda \backslash \sigma_{S}$ is a hyperbolic periodic point of $X$, then $W_{X}^{u}(p) \cap l_{S}=\emptyset$.

Proof. By contradiction, suppose that $W_{X}^{u}(p) \cap l_{S} \neq \emptyset$ for some hyperbolic periodic point $p \in \Lambda \backslash \sigma_{S}$. Since $\sigma_{S}$ is an attracting set by hypothesis, Lemma 1.1 implies that $l_{S}$ is open and closed in $\Lambda \cap S$.

On the other hand, by the previous lemma, there is a connected curve $J^{*} \subset$ $W_{X}^{u}(p) \cap S$ joining $q^{*} \in l_{S}$ to some point $p^{\prime} \in \mathcal{O}_{X}(p) \cap S$. As $\Lambda$ is an attracting set and $p \in \Lambda$, we have $J^{*} \subset \Lambda \cap S$. But, the connectness of $J^{*}$ and the fact that $l_{S}$ is open and closed in $\Lambda \cap S$ imply that $J^{*} \subset l_{S}$. In particular, $p^{\prime} \in l_{S}$ and so $\omega_{X}\left(p^{\prime}\right)=\mathcal{O}_{X}(p) \subset \sigma_{S}$, a contradiction.

To complete the proof of Theorem 1.0 we use the following notation. If $H$ is an invariant set of a vector field $X$, we say that $p \in H$ is nonwandering for $X$ restricted to $H$ if for any neighborhood $U \subset H$ of $p$ and $T>0$ there is $t>T$ so that $X_{t}(U) \cap U \neq \emptyset$. We denote $\Omega(X / H)$ the set of all nonwandering points of $X$ restricted to $H$.

Proof of Theorem 1.0. Let $S$ be a surface transverse to a hyperbolic connected attracting set $\Lambda$ of a vector field $X$. Assume by contradiction that $\sigma_{S}$ is an attracting set. Then, $\sigma_{S} \neq \emptyset$ and so there is $q \in l_{S} \neq \emptyset$ by Lemma 1.2. As $\sigma_{S}$ is an attracting set, we have that $\exists q_{0} \in \alpha_{X}(q) \cap S \neq \emptyset$. The point $q_{0}$ cannot belong to an attracting periodic orbit since $q \in l_{S}$. In particular, $W_{X}^{s}(q) \cap S$ has dimension one. 
Observe that $q_{0} \in \Omega(X / \Lambda)$. Then, by the Shadowing Lemma [PT], there exists a periodic point sequence $p_{n} \in \Lambda$ converging to $q_{0}$. Note that this sequence is not in $\sigma_{S}$ since $q_{0} \notin \sigma_{S}$.

The Local Structure Product [PS] implies that the orbit of $p_{n}$ is neither an attracting nor a repelling periodic orbit for $n$ large since both $p_{n}$ and the backward orbit of $q$ approach $q_{0}$. In particular, $W_{X}^{u}\left(p_{n}\right) \cap S$ has dimension one and there exists $q^{\prime} \in W_{X}^{s}(q) \cap W_{X}^{u}\left(p_{n}\right) \cap S$ for $n$ large enough.

So, for $p=p_{n}$ with $n$ large enough, there is $q^{*} \in W_{X}^{u}(p) \cap l_{S}$ in the positive orbit of $q^{\prime}$ since $q^{\prime}$ is asymptotic to $q \in l_{S}$. This contradicts Corollary 1.4 since $p \in \Lambda \backslash \sigma_{S}$ (for $p_{n} \notin \sigma_{S}$ ) proving the result.

\section{Proof of Theorem A}

The result of this section is

Theorem B. Let $\Lambda$ be a connected hyperbolic attracting set of a three-dimensional vector field with $\operatorname{dim}\left(E^{u}\right)=1$. If $\Lambda$ exhibits a transverse surface $S$ such that $\mathcal{F}_{S}^{u}$ has no closed leaves, then $\sigma_{S}=\emptyset$.

Before its proof we give some examples.

Example 2.1. Let $\Lambda$ be a hyperbolic strange attractor of a three-dimensional vector field. As transitive sets for flows are always connected, we conclude that $\Lambda$ is a connected hyperbolic attracting set of a three-dimensional vector field with $\operatorname{dim}\left(E^{u}\right)=1$. If $\Lambda$ admits a transverse surface $S$ so that $\mathcal{F}_{S}^{u}$ has no closed leaves, the theorem above implies that $\Lambda$ is a suspension because $\sigma_{S}=\emptyset$. This is the content of Theorem A.

Example 2.2. Let $X$ be an Anosov flow defined on a closed 3-manifold $M$. Then, $\Lambda=M$ is obviously a connected hyperbolic attracting set of $X$ with $\operatorname{dim}\left(E^{u}\right)=1$. If $X$ admits a transverse surface $S$ so that the induced unstable foliation $\mathcal{F}_{S}^{u}$ has no closed leaves, then we conclude that $\sigma_{S}=\emptyset$ by the above theorem. In particular, $S$ is a torus and $X$ is a suspension. This result was quoted in [Fe2] as mentioned in $\S 1$.

Example 2.3. Let $\Lambda$ be a suspended hyperbolic attractor of a three-dimensional vector field $X$. Then every transverse surface of $\Lambda$ is a cross-section of it. Indeed, let $S_{0}$ the cross-section of $\Lambda$ and $S$ be a transverse surface of $\Lambda$. We can assume that $\operatorname{dim}\left(E^{u}\right)=1$. Clearly, $\Lambda^{*}=\Lambda \cap S_{0}$ is a hyperbolic attractor of the return map associated to $S_{0}$. If $S$ were not a cross-section, then $\mathcal{F}_{S}^{u}$ would have a closed leave by the above theorem. This closed leave is carried by the flow into a closed leave in $\mathcal{F}_{S_{0}}^{u}$. One concludes that the unstable manifold of $\Lambda^{*}$ would have a closed leave, a contradiction (see the proof of Theorem 8.1 in $[\mathbb{R}]$ ). Thus $\sigma_{S}=\emptyset$ by Theorem A and so $S$ is a cross-section as claimed.

We begin the proof of Theorem B with the following standard fact.

Lemma 2.1. An isolated hyperbolic set without singularities $H$ of a vector field $X$ is an attracting set if $W_{X}^{u}(p) \subset H$ for any $p \in H$ periodic.

Proof. By the Stable Manifold Theorem [HPS], it suffices to show that $U=\{x$ : $\left.\omega_{X}(x) \subset H\right\}$ is a neighborhood of $H$.

For this we proceed as follows. As $H$ is isolated and hyperbolic, $\Omega(X / H)$ is a finite disjoint union $H_{1} \cup \ldots \cup H_{n}$ of basic sets by the Spectral Decomposition 
Theorem [PS]. Remember that a compact invariant set $B$ of $X$ is basic if it is transitive, hyperbolic and isolated. Now, suppose that $\omega_{X}(x) \subset H$. Replacing $x$ by a suitable point in $\omega_{X}(x)$ if necessary, we can assume that $\omega_{X}(x) \subset H_{1}$ (say).

As the periodic points are dense in $H_{1}$, the positive orbit of $x$ passes close to the orbit of some periodic point $p \in H_{1}$. Thus, the orbit of $x$ must be asymptotic to the positive orbit of some point in $W_{X}^{u}(p) \subset H$. This clearly occurs for every point close to $x$ and so $U$ is open as claimed. This completes the proof of the lemma.

Now, let $p$ be a hyperbolic periodic point of a vector field $X$ with $\operatorname{dim}\left(E_{p}^{u}\right)=1$. As already mentioned in the Introduction, there is a strong unstable manifold $W_{X}^{u u}(p)$ passing through $p$ and tangent to $E^{u}$. In particular, $W_{X}^{u u}(p)$ is onedimensional and contained in $W_{X}^{u}(p)$.

Denoting by $t_{p}$ the period of $p$, one has $X_{t_{p}}\left(W_{X}^{u u}(p)\right)=W_{X}^{u u}(p)$. The map $X_{t_{p}} / W_{X}^{u u}(p)$ either preserves or reverses the orientation, and so, $W^{u}(p)$ is either a cylinder or a Moebius band respectively.

A fundamental domain for $W_{X}^{u u}(p)$ is a closed interval $[a, b]^{u u}$ contained in $W_{X}^{u u}(p)$ so that $a \neq p$ and either $X_{t_{p}}(a)=b$ (in the orientation-preserving case) or $X_{2 t_{p}}(a)=b$ (in the orientation-reversing case).

Lemma 2.2. Let $S$ be a transverse surface of a hyperbolic attracting set $\Lambda$ of a vector field $X$ with $\operatorname{dim}\left(E^{u}\right)=1$ and let $p \in \sigma_{S}$ be periodic. Suppose that a fundamental domain $[a, b]^{u u}$ of $W_{X}^{u u}(p)$ satisfies

(1) $X_{t}\left([a, b]^{u u}\right) \cap S=\emptyset$ for all $t \leq 0$ and

(2) every positive orbit of $X$ starting in $[a, b]^{\text {uu }}$ meets $S$.

Then, $\mathcal{F}_{S}^{u}$ has a closed leaf.

Proof. The positive flow of $X$ defines a first intersection map $\Pi$ from $[a, b]^{u u}$ to $S$. As $a$ is in the backward orbit of $b$ and the backward saturation of the domain does not intersect $S$, we conclude that $\Pi(a)=\Pi(b)$. Thus $\Pi\left([a, b]^{u u}\right)$ is a closed curve without self-intersections. By definition, this curve is a leaf of $\mathcal{F}_{S}^{u}$ since it is contained in $W_{X}^{u}(p) \cap S$.

Before the proof of Theorem B let us remember some useful properties of basic sets $B$ for vector fields $X$.

If $B$ is nonsingular, it follows that $B$ is the closure of the periodic orbits of $X$ in $B$. In addition, if $q$ is a point such that $\omega_{X}(q) \subset B$, then there is $x \in B$ such that $q \in W_{X}^{s s}(x)$. In other words, every point asymptotic to $B$ is asymptotic to some point in $B$.

Next we recall the notion of a stable boundary point [NP, [Ch, Definition 2.6]. Let $X$ be a three-dimensional vector field and $B$ a nonsingular basic set of $X$ with $\operatorname{dim}\left(E^{u}\right)=1$. One says that $x \in B$ is a stable boundary point of $B$ if there is an open interval $I \subset W_{X}^{u u}(x)$ containing $x$ such that one of the connected components of $I \backslash\{x\}$ does not intersect $B$.

Proof of Theorem B. Let $S$ be a transverse surface of a connected hyperbolic attracting set $\Lambda$ of $X$ so that $\operatorname{dim}\left(E^{u}\right)=1$. We assume by the proof that $\mathcal{F}_{S}^{u}$ has no closed leaves.

Under such conditions we prove

Lemma 2.3. If $\sigma_{S} \neq \emptyset$, then $\sigma_{S}$ is an attracting set. 
The proof of Theorem B using the above lemma is as follows: Since $\Lambda$ is connected, we conclude that $\sigma_{S}$ cannot be an attracting set by Theorem 1.0. Then, $\sigma_{S}=\emptyset$ by Lemma 2.3 and the proof follows.

We are left to prove Lemma 2.3 in order to prove Theorem B.

Proof of Lemma 2.3. By Lemma 2.1 it is enough to prove that $W_{X}^{u}(p) \subset \sigma_{S}$ for every $p \in \sigma_{S}$ periodic. Recall that $\sigma_{S}$ is nonempty (by hypothesis) isolated and hyperbolic (Lemma 1.1). Moreover, $\Lambda$ has no singularities since it is an attracting set and $\operatorname{dim}\left(E^{u}\right)=1$. In particular, $\sigma_{S}$ has no singularities.

By way of contradiction, assume the existence of $p_{0} \in \sigma_{S}$ periodic and $q_{0} \in$ $W_{X}^{u}\left(p_{0}\right) \backslash \sigma_{S}$. We can assume that $q_{0}$ is a boundary point of a fundamental domain $I=\left[q_{1}, q_{0}\right]^{u u}$ of $W_{X}^{u u}\left(p_{0}\right)$ satisfying $I \cap S=\emptyset$. By definition of fundamental domain, $q_{1}$ is in the backward orbit of $q_{0}$.

As $\Lambda$ is an attracting set, $q_{0} \in \Lambda$. Thus, there is a minimum positive number $t$ so that $X_{t}\left(q_{0}\right) \in S$ because $q_{0} \notin \sigma_{S}$. Then, there is a return map $R$ from $D(R) \subset I$ (the domain of $R$ ) into $S$. Note that both $q_{0}$ and $q_{1}$ belong to $D(R)$ and $R\left(q_{0}\right)=R\left(q_{1}\right)$.

As $\Lambda$ intersects $S$ only in the interior of $S$, we have that $D(R)$ contains a maximal interval $J \subset I$ of the form $J=\left(q_{2}, q_{0}\right]$ for some $q_{1} \leq q_{2}<q_{0}$ (we order the points in $I$ according to the natural interval order).

Observe that $q_{1}<q_{2}$ since, otherwise, $I=J \subset D(R)$ and so the positive orbit of every point in $I$ meets $S$. This would contradict Lemma 2.2 since $\mathcal{F}_{S}^{u}$ has no closed leaves by hypothesis.

Note that $q_{2} \in \sigma_{S}$ for its backward orbit converges to $\mathcal{O}_{X}\left(p_{0}\right)$, the orbit of $p_{0}$, and its forward orbit cannot intersect $S$ since $J$ is maximal. In particular, $\omega_{X}\left(q_{2}\right)$ belongs to a basic set $H$ in the spectral decomposition of $\Omega\left(X / \sigma_{S}\right)$. That such a decomposition exists follows from the fact that $\sigma_{S} \neq \emptyset$ is hyperbolic and isolated (Lemma 1.1).

Now we prove

Claim. There is a periodic orbit $\mathcal{O} \subset H$ of $X$ such that $q_{2} \in W_{X}^{s}(\mathcal{O})$.

Proof of the Claim. As $H$ is a basic set and $\omega_{X}\left(q_{2}\right) \subset H$, we have that $q \in W_{X}^{s s}(x)$ for some $x \in H$. We shall prove that there is $x^{\prime}$ in the positive orbit of $x$ such that $x^{\prime}$ is a stable boundary point of $H$ (in particular $x$ itself is a stable boundary point of $H)$.

To prove this assertion, choose $y \in \omega_{X}(x)$ (thus $y \in H$ ). Let $\Sigma$ be a transverse disc of $X$ such that $y \in \operatorname{int}(\Sigma)$, the interior of $\Sigma$. Choose a sequence $x_{n} \in \operatorname{int}(\Sigma) \cap$ $\mathcal{O}_{X}^{+}(x)$ converging to $y$. Fix $x^{\prime}=x_{n}$ for some $n$ large enough. Denote $F^{s}\left(x^{\prime}\right)$ $\left(\operatorname{resp} . F^{u}\left(x^{\prime}\right)\right)$ the connected component of $W_{X}^{s}\left(x^{\prime}\right) \cap \Sigma\left(\operatorname{resp} . W_{X}^{u}\left(x^{\prime}\right) \cap \Sigma\right)$ containing $x^{\prime}$. As the positive orbits of $q_{2}$ and $x$ come together, one can choose $q_{2}^{\prime} \in \mathcal{O}_{X}^{+}\left(q_{2}\right) \cap$ $F^{s}\left(x^{\prime}\right)$.

By the Tubular Flow-Box Theorem there is a small interval $J^{\prime} \subset J$ (with boundary point $q_{2}$ ) such that the flow of $X$ carries $J^{\prime}$ into an interval $J^{\prime \prime} \subset \operatorname{int}(\Sigma)$ (with boundary point $\left.q_{2}^{\prime}\right)$. Note that the closure of $J^{\prime \prime}$ and $F^{s}\left(x^{\prime}\right)$ intersect transversally at $q_{2}^{\prime}$.

Now we suppose by contradiction that $x^{\prime}$ is not a stable boundary point of $H$. Then, there would exist $z \in H \cap F^{u}\left(x^{\prime}\right)$ arbitrarily close to $x$ in both connected components of $F^{u}\left(x^{\prime}\right) \backslash\left\{x^{\prime}\right\}$. Using the Local Product Structure of $H([\overline{\mathrm{PS}}])$ and the fact that $J^{\prime \prime}$ intersects transversally $F^{s}\left(x^{\prime}\right)$ at $q_{2}^{\prime}$, one could find $z \in H \cap F^{u}\left(x^{\prime}\right)$ 

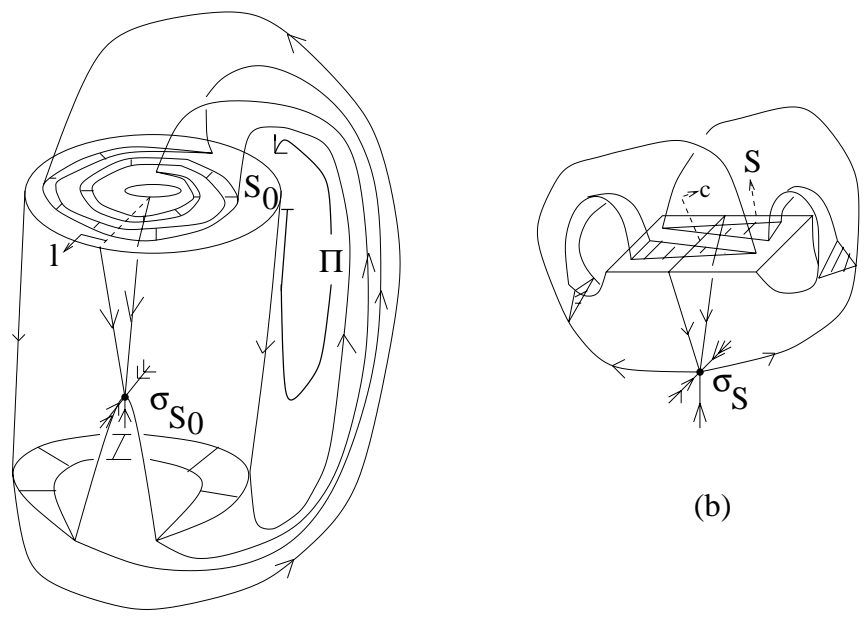

(b)

(a)

FIGURE 2.

such that $W_{X}^{s}(z) \cap J^{\prime \prime} \neq \emptyset$. Taking $w \in W_{X}^{s}(z) \cap J^{\prime \prime} \neq \emptyset$, we have that the positive orbit of $w$ is asymptotic to that of $z \in H$. In particular, $\mathcal{O}_{X}^{+}(w) \cap S=\emptyset$. However, we have that $\mathcal{O}_{X}^{+}(w)$ intersects $S$ by the definition of $J$ (recall that $J^{\prime} \subset J$ and $J^{\prime \prime}$ is in the positive orbit of $J^{\prime}$ ). This contradiction proves that $x^{\prime}$ is a stable boundary point of $H$.

To finish the proof of the Claim we only need to observe that if $B$ is a basic set of a three-dimensional vector field $X$, then every stable boundary point of $B$ belongs to a periodic orbit of $X$ ([NP Proposition 1], [B, Lemme 1.12], [Ch, Lemma 2.7]). In particular, there is a periodic orbit $\mathcal{O} \subset H$ of $X$ such that $x^{\prime} \in W_{X}^{s}(\mathcal{O})$. As $x^{\prime}$ is in the positive orbit of $x$ and $q_{2} \in W_{X}^{s s}(x)$, it follows that $q_{2} \in W_{X}^{s}(\mathcal{O})$. This proves the Claim.

To finish with the proof of Lemma 2.3, choose $J^{\prime} \subset J$ as in the proof of the Claim. Then Inclination-Lemma $[\overline{\mathrm{PT}}]$ implies that there is $x_{0} \in \mathcal{O}$ such that the positive orbit of $J^{\prime}$ approaches a fundamental domain of $W_{X}^{u u}\left(x_{0}\right)$. As the positive orbit of $\operatorname{int}\left(J^{\prime}\right)$ meets $S$, using the stable manifold of $\Lambda$, there is a fundamental domain of $W_{X}^{u u}\left(x_{0}\right)$ (very close to $x_{0}$ ) so that every positive orbit starting in that domain meets $S$. This last fact and Lemma 2.2 contradict once more the assumption that $\mathcal{F}_{S}^{u}$ has no closed leaves. This contradiction completes the proof of Lemma 2.3.

\section{Equivalence of attractors}

In this section we apply $\S 2$ to study the topological equivalence between singular attractors with a transverse surface.

Let us first recall equivalence. Denote $\Lambda$ and $\Lambda^{\prime}$ attractors for the flows $X_{t}$ and $X_{t}^{\prime}$ respectively. We say that $\Lambda$ and $\Lambda^{\prime}$ are equivalent if there are neighborhoods $U$ and $U^{\prime}$ of $\Lambda$ and $\Lambda^{\prime}$ respectively and a homeomorphism $H: U \rightarrow U^{\prime}$ sending positively oriented $X$-orbits into positively oriented $X^{\prime}$-orbits.

The following proposition will be used to study equivalence. 


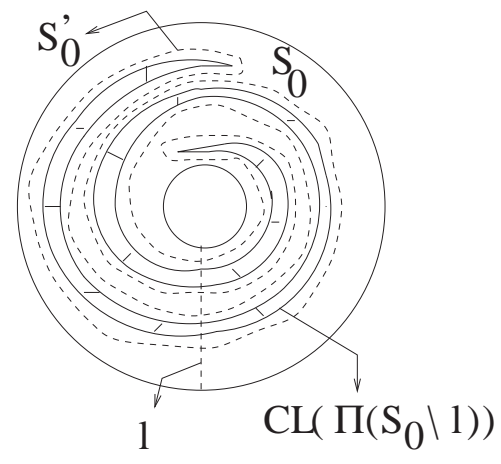

(a)

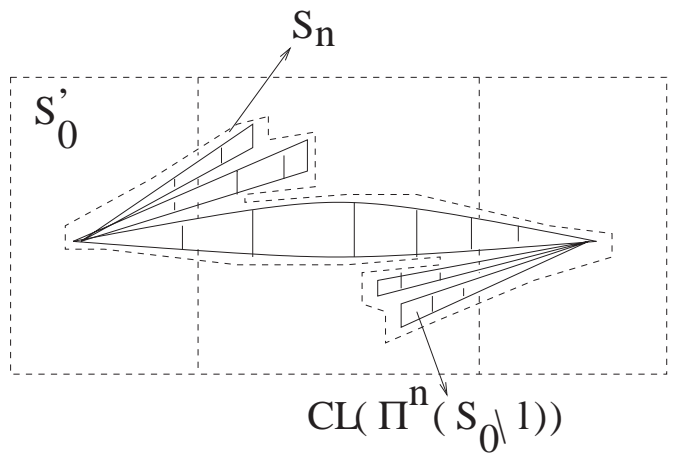

(b)

FIGURE 3.

Proposition 3.1. If two transverse surfaces $S$ and $S^{\prime}$ of an attractor $\Lambda$ satisfy $\sigma_{S}=\sigma_{S^{\prime}}$, then $\Lambda \cap S$ and $\Lambda \cap S^{\prime}$ are homeomorphic.

Proof. Observe that if $q \in \Lambda \cap S$, then $q \notin \sigma_{S}=\sigma_{S^{\prime}}$ and so $X_{\mathbb{R}}(q) \cap S^{\prime} \neq \emptyset$ by the definition of $\sigma_{S^{\prime}}$. In particular, $\forall q \in \Lambda \cap S$ there is $t \in \mathbb{R}$ so that $X_{t}(q) \in S^{\prime}$. This defines a return map $R$ from $\Lambda \cap S$ into $\Lambda \cap S^{\prime}$ which is clearly continuous and one-to-one. Interchanging the roles of $S$ and $S^{\prime}$ in the argument, we obtain a continuous inverse for $R$. Thus $R$ is a homeomorphism, and so, $\Lambda \cap S$ and $\Lambda \cap S^{\prime}$ are homeomorphic finishing the proof.

We are going to consider the examples described in Figure 2. The one in Figure 2 (b) is the well-known geometric Lorenz attractor in [GW]. We denote it by $\Lambda$ and by $X$ its underlying vector field. In this example there is a transverse surface $S$, the top rectangle indicated in that figure. Observe that $\sigma_{S}$ is a singularity (see [GW] for details).

The example in Figure 2(a) comes from the Appendix in [MP]. Here, $S_{0}$ is a two-dimensional annulus and $\sigma_{S_{0}}$ is a singularity as in the previous case. We denote by $\Lambda_{a}$ the attractor of this figure and by $X_{a}$ its underlying vector field.

Also indicated in Figure 2(a) is a return map $\Pi$, induced by the flow of $X_{a}$, from $S_{0} \backslash l$ to the interior of $S_{0}$. Here $l$ denotes a curve contained in the stable manifold of $\sigma_{S_{0}}$. The map $\Pi$ is hyperbolic: it expands along the angular direction and contracts along the radial one. The corresponding stable manifold's quotient in $S_{0}$ is the circle and the corresponding foliation map is transitive, i.e. has a dense orbit.

In Figure 3(a), $S_{0}^{\prime}$ denotes a rectangular region which bounds $C L\left(\Pi\left(S_{0} \backslash l\right)\right)$. Figure 3(b) describes $S_{0}^{\prime}$ in a precise way. There, $S_{n}$ denotes a connected region which bounds the closure $C L\left(\Pi^{n}\left(S_{0} \backslash l\right)\right)$ of the successive $\Pi$-iterates of $S_{0} \backslash l, n \in \mathbb{N}$. We can choose $S_{n}$ arbitrarily close to $\Lambda_{a} \cap S_{0}$ as such iterates converge to $\Lambda_{a} \cap S_{0}$ in the Hausdorff topology.

Summarizing, we have the following properties for $S_{n}$ :

1. $S_{0} \cap \Lambda_{a}=S_{n} \cap \Lambda_{a}$, 
2. $S_{n} \subset S_{n-1}$, for all $n$, and

3. $S_{n}$ converges in the Hausdorff topology to $\Lambda_{a} \cap S_{0}$ as $n \rightarrow \infty$.

Let us see how these properties and Proposition 3.1 imply

Theorem 3.2. $\Lambda_{a}$ and $\Lambda$ are not equivalent.

As mentioned in $\S 1$ this result is claimed in $[\mathrm{MP}$, but the proof there is just a sketch. The following proof fills in details of the proof in [MP] using the ideas developed in $\S 2$.

Proof. By Property (1) above we have that $\sigma_{S_{0}}=\sigma_{S_{n}}$ for all $n$. In particular, we can replace $S_{0}$ by $S_{n}$ so that $\sigma_{S_{n}}$ is still $\sigma_{S_{0}}$.

By way of contradiction, assume that there is an equivalence $H: U_{a} \rightarrow U$ from fixed neighborhoods $U_{a}$ and $U$ of $\Lambda_{a}$ and $\Lambda$ respectively. Then, $H\left(\Lambda_{a}\right)=\Lambda$ since $\Lambda$ has no proper attractors and $H$ is an equivalence.

Choosing $n$ large, by Property (3), we can assume that $S_{n} \subset U_{a}$. Thus $S^{\prime}=$ $H\left(S_{n}\right)$ is well-defined. Using the equivalence, since $S_{n}$ is a transverse surface of $\Lambda_{a}$, there is an open covering $\mathcal{U}$ of $S^{\prime}$ so that every orbit of $X$ in $\mathcal{U}$ intersects $S^{\prime}$ only at one point. Then, we can modify $S^{\prime}$ using the flow of $X$ to obtain a transverse surface of $H\left(\Lambda_{a}\right)=\Lambda$ still denoted by $S^{\prime}$. Observe that $\sigma_{S^{\prime}}=\sigma_{S}$ since $H$ is an equivalence.

We conclude that $\Lambda \cap S^{\prime}$ and $\Lambda \cap S$ are homeomorphic by Proposition 3.1. So, $\Lambda_{a} \cap S_{n}=H^{-1}\left(\Lambda \cap S^{\prime}\right)$ and $\Lambda \cap S$ are homeomorphic since $H$ is a homeomorphism.

On the other hand, $\Lambda_{a} \cap S_{n}$ is a connected set. This follows from Property (1) together with the fact that every $S_{n}$ is connected, the sequence $S_{n}$ is nested (by Property (2)), and $\bigcap_{n} S_{n}=\Lambda_{a} \cap S_{0}$ (by Property (3)).

In particular, $\Lambda \cap S$ is connected since it is homeomorphic to $\Lambda_{a} \cap S_{n}$. But this is a contradiction since it is easy to check in Figure 2(b) that $\Lambda \cap S$ is not connected. Indeed, this set is separated by the two triangles depicted inside the top rectangle $S$ in that figure.

We conclude that $\Lambda$ and $\Lambda_{a}$ cannot be equivalent, proving the result.

\section{REFERENCES}

[B] Beguin, F., Champs de vecteurs hyperboliques en dimension 3, Thése Université de Bourgogne (1999).

[BW] Birman, J., Williams, R., Knotted periodic orbits in dynamical systems II : Knot holders for fibered knots, Low-dimensional Topology, Contemp. Math. 20, Amer. Math. Soc., Providence, R. I., (1983), 1-60. MR 86a:58084

[BL] Bonatti, C., Langevin, R., Un example de flot d'Anosov transitif transverse à un tore et non conjugué à une suspension, Ergod. Theory Dynam. Sys. 14 (1994), 633-643. MR 95j:58129

[Bo] Bowen, R., Mixing Anosov flows, Topology 15 (1976), 77-79. MR 52:11999

[Ch] Christy, J., Intransitive Anosov Flows on three-manifolds, Thesis, Berkeley (1984).

[Fe1] Fenley, S., Surface transverse to pseudo-Anosov flows and virtual fiber in 3-manifolds, Topology 38 (1999), 823-859. MR 2001a:57041

[Fe2] Fenley, S., Incompressible tori transverse to Anosov flows in 3-manifolds, Ergod. Theory Dynam. Sys. 17 (1997), 105-121. MR 98c:58122

[Fr] Fried, D., The geometry of cross sections to flows, Topology 21 (1982), 353-371. MR 84d:58068

[GW] Guckenheimer, J., Williams, R.F., Structural stability of Lorenz attractor, Publ. Math. IHES 50 (1979), 59-72. MR 82b:58055a

[HPS] Hirsch, M., Pugh, C., Shub, M., Invariant manifolds, Lecture Notes in Math. 583 (1977), Springer-Verlag. MR 58:18595 
[NP] Newhouse, S., Palis, J., Hyperbolic nonwandering sets on two-dimensional manifolds, Dynamical Systems, Peixoto (Ed.), Salvador, (1973), 293-301. MR 49:4044

[MP] Morales, C. A., Pujals, E. R., Singular strange attractors on the boundary of MorseSmale systems, Ann. Ec. Norm. Sup., 30 (1997), 693- 717. MR 98k:58137

[PT] Palis, J., Takens, F., Hyperbolicity and sensitive chaotic dynamics at homoclinic bifurcations, (1993), Cambridge Univ. Press. MR 94h:58129

[PS] Pugh, C., Shub, M., The $\Omega$-stability theorem for flows, Inventiones Math. 11 (1970), 150-158. MR 44:4782

[R] Robinson, C., Dynamical Systems, Stability, symbolic dynamics, and chaos, Studies in Advanced Mathematics CRC Press, Boca Raton, FL (1995). MR 97e:58064

Instituto de Matematica, Universidade Federal do Rio de Janeiro, C. P. 68.530, CEP 21.945-970, Rio DE JANEIRO, BRAZIL

Current address: Universidad Centroccidental Lizandro Alvarado, Departamento de Matemática, Decamato de Ciencias, Apdo 400, Barquisimeto, Venezuela

E-mail address: wilmerc@pg.im.ufrj.br

Instituto de Matematica, Universidade Federal do Rio de Janeiro, C. P. 68.530, CeP 21.945-970, Rio DE JANEIRo, BRAZIL

E-mail address: morales@impa.br 